\title{
ASYMPTOTIC STUDY OF MIXED ROTATING MHD SYSTEM
}

\author{
RIDHA SELmI
}

\begin{abstract}
Asymptotic behavior of three-dimensional mixed, periodic and rotating magnetohydrodynamic system is investigated as the Rossby number goes to zero. The system presents the difficulty to be singular and mixed, that is hyperbolic in the vertical direction and parabolic in the horizontal one. The divergence free condition and the spectral properties of the penalization operator are crucial in the proofs. The main tools are the energy method, the Schochet's method and products laws in anisotropic Sobolev spaces.
\end{abstract}

\section{Introduction}

In this paper, we deal with the asymptotic study of the following incompressible, rotating and anisotropic magnetohydrodynamic model denoted by $\left(\mathrm{MHD}^{\varepsilon}\right)$ :

$$
\left\{\begin{array}{l}
\partial_{t} u-\nu \Delta_{h} u+u \cdot \nabla u-\operatorname{curl} b \times b+\frac{1}{\varepsilon} \operatorname{curl} b \times e_{2}+\frac{1}{\varepsilon} u \times e_{3}=-\nabla p \text { in } \mathbb{R}^{+} \times \mathbb{T}^{3} \\
\partial_{t} b-\eta \Delta_{h} b+u \cdot \nabla b-b \cdot \nabla u+\frac{1}{\varepsilon} \operatorname{curl}\left(u \times e_{2}\right)=0 \text { in } \mathbb{R}^{+} \times \mathbb{T}^{3} \\
\operatorname{div} u=0 \text { in } \mathbb{R}^{+} \times \mathbb{T}^{3} \\
\operatorname{div} b=0 \text { in } \mathbb{R}^{+} \times \mathbb{T}^{3} \\
\left.(u, b)\right|_{t=0}=\left(u_{0}, b_{0}\right) \text { in } \mathbb{T}^{3},
\end{array}\right.
$$

where the velocity field $u$, the induced magnetic perturbation $b$ and the pressure $p$ are unknown functions of time $t$ and space variable $x=\left(x_{1}, x_{2}, x_{3}\right)=\left(x_{h}, x_{3}\right)$, the torus $\mathbb{T}^{3}$ denotes the unit periodic box in $\mathbb{R}^{3}$ defined by

$$
\mathbb{T}^{3}:=\Pi_{j=1}^{3} \mathbb{R} / \mathbb{Z},
$$

$e_{2}$ and $e_{3}$ are the second and the third vector of the Cartesian coordinate system, $\nu$ and $\eta$ designate respectively the dynamic viscosity and the magnetic diffusivity and $\varepsilon$ is the Rossby number destined to tend to zero and defined as the ratio between the fluid's typical velocity to the earth rotation velocity

Received February 23, 2008; Revised May 16, 2008.

2000 Mathematics Subject Classification. 35B10, 35B25, 35B40.

Key words and phrases. MHD system, hyperbolic-parabolic system, anisotropic Sobolev spaces, divergence free condition, asymptotic behavior, Schocht's methods. 
around the axis $e_{3}$. Here, $\Delta_{h}$ denotes the horizontal Laplace operator given by $\Delta_{h}=\partial_{1}^{2}+\partial_{2}^{2}$.

About physical motivation, as it is stated in [3], MHD systems model the magnetohydrodynamic flow in the Earth's core which is believed to support a self exited dynamo process generating the Earth's magnetic field. However, the system $\left(\mathrm{MHD}^{\varepsilon}\right)$ we are considering in this paper is just a mathematical model. As it is detailed in [3, 4], MHD systems are a coupling between fluid equations and Maxwell's equations where the electrical field can be expressed as a function of the magnetic one via the induction equation.

Applying $\mathbb{P}$, the $L^{2}\left(\mathbb{T}^{3}\right)$-orthogonal projection onto divergence free vector fields, to the first equation of $\left(\mathrm{MHD}^{\varepsilon}\right)$, one sees that $U=(u, b)$ satisfies the following abstract system:

$$
\left(\mathcal{S}^{\varepsilon}\right)\left\{\begin{array}{l}
\partial_{t} U+Q(U, U)+a_{2}(D) U+L^{\varepsilon}(U)=0 \text { in } \mathbb{R}^{+} \times \mathbb{T}^{3} \\
\operatorname{div} u=0 \text { in } \mathbb{R}^{+} \times \mathbb{T}^{3} \\
\operatorname{div} b=0 \text { in } \mathbb{R}^{+} \times \mathbb{T}^{3} \\
\left.U\right|_{t=0}=U_{0} \text { in } \mathbb{T}^{3}
\end{array}\right.
$$

where, after simplification [1], the quadratic term $Q$ is defined by

$$
Q(U, U)=\left(\begin{array}{c}
\mathbb{P}(u \cdot \nabla u)-\mathbb{P}(b \cdot \nabla b) \\
u \cdot \nabla b-b \cdot \nabla u
\end{array}\right),
$$

the viscous term is

$$
a_{2}(D) U=\left(-\nu \Delta_{h} u,-\eta \Delta_{h} b\right)
$$

and the linear perturbation $L^{\varepsilon}$ is given by

$$
L^{\varepsilon}(U)=\frac{1}{\varepsilon} L(U)=\frac{1}{\varepsilon}\left(\begin{array}{c}
\partial_{2} b+\mathbb{P}\left(u \times e_{3}\right) \\
\partial_{2} u
\end{array}\right) .
$$

We note that $L^{\varepsilon}$ is a skew-symmetric linear operator. This skew-symmetry is an important property for several reasons. Indeed, the singular perturbation disappears while using energy methods in the proof of the existence and uniqueness results. Moreover, such skew-symmetry is a necessary condition to apply the Schochet's method, a fact which will be detailed below.

To announce the mathematical results, we begin by introducing a kind of functional spaces which are well suited for anisotropic systems such as $\left(\mathrm{MHD}^{\varepsilon}\right)$. For any real number $s$ and $s^{\prime}$ the anisotropic Sobolev space $H^{s, s^{\prime}}\left(\mathbb{T}^{3}\right)$ is the space with Sobolev regularity $H^{s}$ in $x_{h}=\left(x_{1}, x_{2}\right)$ and $H^{s^{\prime}}$ in $x_{3}$. That is the tempered distributions $f$ satisfying

$$
\|f\|_{H^{s, s^{\prime}}}:=\left\|\left(1+\left|k_{h}\right|^{2}\right)^{\frac{s}{2}}\left(1+\left|k_{3}\right|^{2}\right)^{\frac{s^{\prime}}{2}} \mathcal{F}(f)\right\|_{L^{2}}<\infty,
$$

where $\mathcal{F}$ designates the Fourier transform and $k_{h}=\left(k_{1}, k_{2}\right)$.

As in our previous work ([2]), we prove the following theorem:

Theorem 1. Let $s>\frac{1}{2}$ be a real number and $U_{0}=\left(u_{0}, b_{0}\right) \in H^{0, s}\left(\mathbb{T}^{3}\right)$, such that $\operatorname{div} u_{0}=0$ and $\operatorname{div} b_{0}=0$. There exists a positive time $T$ such that for all 
$\varepsilon>0$, there exists a unique solution $U^{\varepsilon}$ of $\left(\mathcal{S}^{\varepsilon}\right)$ satisfying $U^{\varepsilon} \in C_{T}^{0}\left(H^{0, s}\left(\mathbb{T}^{3}\right)\right)$ and $\nabla_{h} U^{\varepsilon} \in L_{T}^{2}\left(H^{0, s}\left(\mathbb{T}^{3}\right)\right)$.

Moreover, for all $t \leq T, U^{\varepsilon}$ satisfies the following energy estimate:

(1) $\left\|U^{\varepsilon}(t, \cdot)\right\|_{H^{0, s}\left(\mathbb{T}^{3}\right)}^{2}+\min (\nu, \eta) \int_{0}^{t}\left\|\nabla_{h} U^{\varepsilon}(\tau, \cdot)\right\|_{H^{0, s}\left(\mathbb{T}^{3}\right)}^{2} d \tau \leq\left\|U_{0}\right\|_{H^{0, s}\left(\mathbb{T}^{3}\right)}^{2}$.

Furthermore, there exists a constant c such that if

$$
\left\|U_{0}\right\|_{H^{0, s}\left(\mathbb{T}^{3}\right)} \leq c \min (\nu, \eta),
$$

then the solution is global.

The proof is almost similar to the ones given in [2]. In fact, Lemma 1 and Lemma 4 stated there in the case of $\mathbb{R}^{3}$, still apply in the case of the tore $\mathbb{T}^{3}$ and are the bases of the existence and uniqueness results. Especially, about Lemma 4 , we note the basic fact that if any integral converges in $+\infty$, its corresponding series has the same fate.

The aim of this work is to focus on the asymptotic behavior of the unique solution, as the Rossby number $\varepsilon$ goes to zero. To do so, let $\left(U^{\varepsilon}\right)$ be the family of strong solutions of the system $\left(\mathcal{S}^{\varepsilon}\right)$ with initial data $U_{0}$ given by Theorem 1 . It is clear that when trying to take the limit when $\varepsilon \rightarrow 0$, the classical proofs (for example [11], [14]) no longer work because $\left(\partial_{t} U^{\varepsilon}\right)$ is not bounded with respect to $\varepsilon$. To overcome this difficulty, we use the method introduced by S. Schochet in [13]. This can be done since the penalization operator $L^{\varepsilon}$ is skew symmetric. Such procedure consists in filtering the system by the group $\mathcal{L}(t)$ associated to $L^{\varepsilon}$. It allows to obtain a new system called, in the literature, a filtered system, where the penalized part disappears. Thus, it will be possible to look, in the sense of distributions, for the limit system satisfied by the eventual limit $V$ of the filtered solution $V^{\varepsilon}$ given by $V^{\varepsilon}:=\mathcal{L}\left(-\frac{t}{\varepsilon}\right) U^{\varepsilon}$. Namely, we establish the following convergence result:

Theorem 2. Let $s>\frac{1}{2}$ and $U_{0}=\left(u_{0}, b_{0}\right) \in H^{0, s}\left(\mathbb{T}^{3}\right)$ such that $\operatorname{div} u_{0}=$ $\operatorname{div} b_{0}=0$. Let $U^{\varepsilon}=\left(u^{\varepsilon}, b^{\varepsilon}\right)$ be the family of solutions of $\left(\mathcal{S}^{\varepsilon}\right)$ given by Theorem 1. Then, for all $s^{\prime}<s$, the family $V^{\varepsilon}=\left(v^{\varepsilon}, c^{\varepsilon}\right)$ converges strongly in $C_{T}^{0}\left(H^{0, s^{\prime}}\right)$ to the solution $V$ of the following limit system:

$$
(L S)\left\{\begin{array}{l}
\partial_{t} V+Q^{0}(V, V)+a_{2}(D) V=0 \text { in }[0, T] \times \mathbb{T}^{3} \\
\operatorname{div} v=0 \text { in }[0, T] \times \mathbb{T}^{3} \\
\operatorname{div} c=0 \text { in }[0, T] \times \mathbb{T}^{3} \\
\left.V\right|_{t=0}=U_{0}=\left(u_{0}, b_{0}\right) \text { in } \mathbb{T}^{3},
\end{array}\right.
$$

where we set, in the sense of distributions,

$$
Q^{0}(V, V):=\lim _{\varepsilon \rightarrow 0} Q^{\varepsilon}(V, V)
$$

Here, since we will focus on justifying a local in time convergence result, $T$ is any uniform time of existence of both systems $\left(\mathcal{S}^{\varepsilon}\right)$ and $(L S)$.

By taking the limit as $\varepsilon \rightarrow 0$, we want to approximate the solution of $\left(\mathrm{MHD}^{\varepsilon}\right)$ when $\varepsilon$ is very small with the solution $V$ of $(L S)$. According to $[4,5]$, this limit 
is relevant to the Earth's core which is believed to be in the asymptotic regime of the small Rossby number $\left(\varepsilon \sim 10^{-7}\right)$, so that this approximation works well.

Contrarily to our previous work $([12])$ and references therein, where a large Sobolev exponent was needed to establish convergence results, the main convergence result here is proved in the space $H^{0, s^{\prime}}$ for any $s^{\prime}<s$. This is done thanks to the divergence free condition and some anisotropic product laws introduced in [10]. In fact, the system is Euler type in the vertical direction so such condition allows us to fill the gap in the regularity caused by the lack of the operator $\partial_{3}^{2}$. The fundamental idea is to use $\partial_{3} U_{3}=-\operatorname{div}_{h} U_{h}$, and to infer that the solution is more regular, with respect to the vertical variable $x_{3}$, then expected. Such regularity is disposed in the system by the horizontal diffusion process. Certainly, this needs some extra technicalities like commutator inequalities and properties of the projection $\mathbb{P}$. The proof uses energy method and estimations are done in $H^{0,-\frac{1}{2}}$ because of the presence of terms like $w_{3} \partial_{3} v_{j}$ for $1 \leq j \leq 2$. Also, some decomposition into high frequencies part and low frequencies part of the forcing term is dictated. Indeed, those terms converge weakly to zero but not strongly; a matter which is undesirable while applying Gronwall Lemma.

\section{Convergence result}

\subsection{Study of the limit system}

We begin by considering the following "wave equation":

$$
\left\{\begin{array}{l}
\partial_{t} U+L(U)=0 \text { in } \mathbb{R}^{+} \times \mathbb{T}^{3} \\
\operatorname{div} u=0 \text { in } \mathbb{R}^{+} \times \mathbb{T}^{3} \\
\operatorname{div} b=0 \text { in } \mathbb{R}^{+} \times \mathbb{T}^{3} \\
U(0)=U_{0} \text { in } \mathbb{T}^{3}
\end{array}\right.
$$

where $L$ is the operator defined by

$$
\left(\begin{array}{c}
\partial_{2} b+\mathbb{P}\left(u \times e_{3}\right) \\
\partial_{2} u
\end{array}\right) .
$$

Denote by $\mathcal{L}$ its associate evolution equation group. $\mathcal{L}(t)$ is explicitly given by (7) and formally

$$
\mathcal{L}(t)=\exp (-t L)
$$

Moreover, using the skew-symmetry of $L$, classical $L^{2}$-scalar product computation leads to

$$
{ }^{t} \mathcal{L}(t)=\mathcal{L}(-t)
$$

For the above "wave equation" system, we prove the following lemma:

Lemma 1. The above system has a global solution denoted by

$$
U(t)=\mathcal{L}(t) U_{0},
$$

such that for all $s$ and $s^{\prime} \in \mathbb{R}$, and for all $U_{0}$ in $H^{s, s^{\prime}}\left(\mathbb{T}^{3}\right)$,

$$
\left\|\mathcal{L}(t) U_{0}\right\|_{H^{s, s^{\prime}\left(\mathbb{T}^{3}\right)}}=\left\|U_{0}\right\|_{H^{s, s^{\prime}}\left(\mathbb{T}^{3}\right)}=\left\|{ }^{t} \mathcal{L}(t) U_{0}\right\|_{H^{s, s^{\prime}}\left(\mathbb{T}^{3}\right)} .
$$


As it will appear in the proof, equality (3) is due to the skew-symmetry of the operator $L$ and it is the main tools for the proof of our convergence result. In fact, thanks to this equality estimates on anisotropic Sobolev norms of filtered solution $V^{\varepsilon}$ applies for the anisotropic Sobolev norms of $U^{\varepsilon}$. An elementary model is the following: Consider the differential equation

$$
(E): \quad \frac{d u}{d t}=f(u)+\frac{i}{\varepsilon} u,
$$

multiplying $(E)$ by $e^{-\frac{i}{\varepsilon} t}$ and putting $v=e^{-\frac{i}{\varepsilon} t} u$, then $(E)$ will be equivalent to

$$
\left(E^{\prime}\right): \quad \frac{d v}{d t}=e^{-\frac{i}{\varepsilon} t} f\left(e^{\frac{i}{\varepsilon} t} v\right) .
$$

In this way, we note the two important facts: the first is that the singular perturbation which prevent us to take the limit directly in the system will disappear; the second is that $|v|=|u|$ so that estimations obtained for $|v|$ holds for $|u|$.

Proof of Lemma 1. The system (2) is equivalent to

$$
\left\{\begin{array}{l}
\partial_{t} u+\mathbb{P}\left(u \times e_{3}\right)+\partial_{2} b=0 \text { in } \mathbb{R}^{+} \times \mathbb{T}^{3} \\
\partial_{t} b+\partial_{2} u=0 \text { in } \mathbb{R}^{+} \times \mathbb{T}^{3} \\
\operatorname{div} u=0 \text { in } \mathbb{R}^{+} \times \mathbb{T}^{3} \\
\operatorname{div} b=0 \text { in } \mathbb{R}^{+} \times \mathbb{T}^{3} \\
U(0)=U_{0} \text { in } \mathbb{T}^{3}
\end{array}\right.
$$

If we apply the operator "curl" to the first equation, then by the partial Fourier transform with respect to the space variable, we get

$$
\left\{\begin{array}{l}
\partial_{t} i M(k) \hat{u}(t, k)+i k_{3} \hat{u}(t, k)-k_{2} M(k) \hat{b}(t, k)=0 \text { in } \mathbb{R}^{+} \times \mathbb{Z}^{3} \\
\partial_{t} \hat{b}(t, k)+i k_{2} \hat{u}(t, k) \text { in } \mathbb{R}^{+} \times \mathbb{Z}^{3} \\
k \cdot \hat{u}=0 \text { in } \mathbb{R}^{+} \times \mathbb{Z}^{3} \\
k \cdot \hat{b}=0 \text { in } \mathbb{R}^{+} \times \mathbb{Z}^{3} \\
\hat{U}(0, k)=\hat{U}_{0}(k) \text { in } \mathbb{Z}^{3}
\end{array}\right.
$$

where

$$
M(k)=\left(\begin{array}{ccc}
0 & -k_{3} & k_{2} \\
k_{3} & 0 & -k_{1} \\
-k_{2} & k_{1} & 0
\end{array}\right) .
$$

We recall that the eigenvalues of $M(k)$ are $\pm i|k|$ and 0 . The corresponding eigenvectors are $\rho(k)^{ \pm}$and $\rho(k)^{0}=\frac{k}{|k|}$ which is not divergence free. Vectors $\rho(k)^{ \pm}$are respectively given by

$$
\rho(k)^{+}=\left\{\begin{array}{l}
\frac{\sqrt{2}}{2}\left(1,-i \frac{k_{3}}{\left|k_{3}\right|}, 0\right) \quad \text { if } \quad k_{1}^{2}+k_{2}^{2}=0, \\
\frac{(\alpha(k), \beta(k), \gamma(k))}{\left(|\alpha(k)|^{2}+|\beta(k)|^{2}+|\gamma(k)|^{2}\right)^{\frac{1}{2}}} \quad \text { if } \quad k_{1}^{2}+k_{2}^{2} \neq 0,
\end{array}\right.
$$


where

$$
(\alpha(k), \beta(k), \gamma(k))=\left(-k_{1} k_{3}-i k_{2}|k|,-k_{2} k_{3}+i k_{1}|k|, k_{1}^{2}+k_{2}^{2}\right)
$$

and

$$
\rho(k)^{-}=\overline{\rho(k)^{+}} .
$$

In this eigenbase, the velocity field $u$ and the magnetic perturbation $b$ can be expressed as follows:

$$
\hat{u}(t, k)=\hat{u}^{+}(t, k) \rho(k)^{+}+\hat{u}^{-}(t, k) \rho(k)^{-}
$$

and

$$
\hat{b}(t, k)=\hat{b}^{+}(t, k) \rho(k)^{+}+\hat{b}^{-}(t, k) \rho(k)^{-} .
$$

In term of variables $\hat{u}^{+}, \hat{u}^{-}, \hat{b}^{+}$and $\hat{b}^{-}$, system (5) splits into four equations summarized in the following system

$$
\left\{\begin{array}{l}
\partial_{t} \hat{U}^{ \pm}(t, k)+A^{ \pm}(k) \hat{U}^{ \pm}(t, k)=0 \text { in } \mathbb{R}^{+} \times \mathbb{T}^{3} \\
\hat{U}^{ \pm}(0, k)=\left(\hat{u}^{ \pm}(0, k), \hat{b}^{ \pm}(0, k)\right) \text { in } \mathbb{T}^{3}
\end{array}\right.
$$

where

$$
\hat{U}^{ \pm}=\left(\hat{u}^{ \pm}, \hat{b}^{ \pm}\right)
$$

and

$$
A^{ \pm}(k)=\left(\begin{array}{cc}
\mp i \frac{k_{3}}{|k|} & \pm i k_{2} \\
\pm i k_{2} & 0
\end{array}\right)
$$

Here we note that

$$
\hat{u}^{0}(t, k)=\left\langle\hat{u}(t, k) \mid \rho(k)^{0}\right\rangle=0
$$

because $\operatorname{div} u=0$ which reads in frequency space

$$
k_{1} \hat{u}_{1}+k_{2} \hat{u}_{2}+k_{3} \hat{u}_{3}=0
$$

and so does $\hat{b}^{0}(t, k)$. Let

$$
A=\operatorname{diag}\left(A^{+}, A^{-}\right) .
$$

The eigenvalues of $A$ are $\omega_{1}, \omega_{2}, \omega_{3}$ and $\omega_{4}$ defined by

$$
\begin{aligned}
\omega_{1}(k) & =\frac{i}{2|k|}\left(-k_{3}+\sqrt{k_{3}^{2}+4 k_{2}^{2}|k|^{2}}\right), \\
\omega_{2}(k) & =\frac{i}{2|k|}\left(-k_{3}-\sqrt{k_{3}^{2}+4 k_{2}^{2}|k|^{2}}\right), \\
\omega_{3}(k) & =\frac{i}{2|k|}\left(k_{3}+\sqrt{k_{3}^{2}+4 k_{2}^{2}|k|^{2}}\right)
\end{aligned}
$$

and

$$
\omega_{4}(k)=\frac{i}{2|k|}\left(k_{3}-\sqrt{k_{3}^{2}+4 k_{2}^{2}|k|^{2}}\right) .
$$


If we denote by $\varrho_{1}, \varrho_{2}, \varrho_{3}$ and $\varrho_{4}$ the corresponding eigenvectors, $U$ will be explicitly given by

$$
\mathcal{F}(U)(t, k)=\sum_{1 \leq j \leq 4} \exp \left(\omega_{j}(k) t\right)\left\langle\mathcal{F}(U)(0, k) \mid \varrho_{j}(k)\right\rangle \varrho_{j}(k) .
$$

Then,

$$
\begin{aligned}
U(t, k) & =\mathcal{F}^{-1}\left(\sum_{1 \leq j \leq 4} \exp \left(\omega_{j}(k) t\right)\left\langle\mathcal{F}\left(U_{0}\right)(k) \mid \varrho_{j}(k)\right\rangle \varrho_{j}(k)\right) \\
& =\mathcal{L}(t) U_{0} .
\end{aligned}
$$

For our case, system $\left(\mathcal{S}^{\varepsilon}\right)$ deals with the operator

$$
L^{\varepsilon}(U)=\frac{1}{\varepsilon} L(U)=\frac{1}{\varepsilon}\left(\begin{array}{c}
\partial_{2} b+\mathbb{P}\left(u \times e_{3}\right) \\
\partial_{2} u
\end{array}\right) .
$$

So, if we define $V^{\varepsilon}$ by

$$
V^{\varepsilon}(t, x):=\mathcal{L}\left(-\frac{t}{\varepsilon}\right) U^{\varepsilon}(t, x)=\left(v^{\varepsilon}, c^{\varepsilon}\right),
$$

then

$$
V^{\varepsilon}(0, x)=U^{\varepsilon}(0, x)=U_{0}
$$

and

$$
\mathcal{F}\left(V^{\varepsilon}\right)(t, k)=\sum_{1 \leq j \leq 4} \exp \left(-\omega_{j}(k) \frac{t}{\varepsilon}\right)\left\langle\mathcal{F}\left(U_{0}\right)(k) \mid \varrho_{j}(k)\right\rangle \varrho_{j}(k) .
$$

$V^{\varepsilon}$ satisfies the following system:

$$
\left(\tilde{\mathcal{S}}^{\varepsilon}\right)\left\{\begin{array}{l}
\partial_{t} V^{\varepsilon}+Q^{\varepsilon}\left(V^{\varepsilon}, V^{\varepsilon}\right)+a_{2}(D) V^{\varepsilon}=0 \text { in } \mathbb{R}^{+}=\times \mathbb{T}^{3} \\
\operatorname{div} v^{\varepsilon}=0 \text { in } \mathbb{R}^{+} \times \mathbb{T}^{3} \\
\operatorname{div} c^{\varepsilon}=0 \text { in } \mathbb{R}^{+} \times \mathbb{T}^{3} \\
\left.V^{\varepsilon}\right|_{t=0}=U_{0}=\left(u_{0}, b_{0}\right) \text { in } \mathbb{T}^{3},
\end{array}\right.
$$

where the filtered quadratic form $Q^{\varepsilon}$ is given by

$$
Q^{\varepsilon}\left(V^{\varepsilon}, V^{\varepsilon}\right)=\mathcal{L}\left(-\frac{t}{\varepsilon}\right) \mathbb{P} Q^{\varepsilon}\left(\mathcal{L}\left(\frac{t}{\varepsilon}\right) V^{\varepsilon}, \mathcal{L}\left(\frac{t}{\varepsilon}\right) V^{\varepsilon}\right) .
$$

In Fourier variables,

$$
2 \mathcal{F}\left(Q^{\varepsilon}\left(V_{1}^{\varepsilon}, V_{2}^{\varepsilon}\right)\right)(n)=\left(\begin{array}{c}
a_{11} \\
a_{21}
\end{array}\right)
$$

where

$$
\begin{aligned}
a_{11}= & \sum_{1 \leq j, j^{\prime}, j^{\prime \prime} \leq 4} \sum_{k+m=n} e^{-i \frac{t}{\varepsilon} \omega_{k, m, n}^{j, j^{\prime}, j^{\prime \prime}}} P(n)\left\langle\left(v_{1}^{j^{\prime}}(k) m v_{2}^{j^{\prime \prime}}(m)\right) \mid \varrho^{j}(n)\right\rangle \varrho^{j}(n) \\
& +\sum_{1 \leq j, j^{\prime}, j^{\prime \prime} \leq 4} \sum_{k+m=n} e^{-i \frac{t}{\varepsilon} \omega_{k, m, n}^{j, j^{\prime}, j^{\prime \prime}}} P(n)\left\langle\left(v_{2}^{j^{\prime}}(k) m v_{1}^{j^{\prime \prime}}(m)\right) \mid \varrho^{j}(n)\right\rangle \varrho^{j}(n) \\
& -\sum_{1 \leq j, j^{\prime}, j^{\prime \prime} \leq 4} \sum_{k+m=n} e^{-i \frac{t}{\varepsilon} \omega_{k, m, n}^{j, j^{\prime}, j^{\prime \prime}}} P(n)\left\langle\left(c_{1}^{j^{\prime}}(k) m c_{2}^{j^{\prime \prime}}(m)\right) \mid \varrho^{j}(n)\right\rangle \varrho^{j}(n)
\end{aligned}
$$




$$
-\sum_{1 \leq j, j^{\prime}, j^{\prime \prime} \leq 4} \sum_{k+m=n} e^{-i \frac{t}{\varepsilon} \omega_{k, m, n}^{j, j^{\prime}, j^{\prime \prime}}} P(n)\left\langle\left(c_{2}^{j^{\prime}}(k) m c_{1}^{j^{\prime \prime}}(m)\right) \mid \varrho^{j}(n)\right\rangle \varrho^{j}(n)
$$

and

$$
\begin{aligned}
a_{21}= & \sum_{1 \leq j, j^{\prime}, j^{\prime \prime} \leq 4} \sum_{k+m=n} e^{-i \frac{t}{\varepsilon} \omega_{k, m, n}^{j, j^{\prime}, j^{\prime \prime}}}\left\langle\left(v_{1}^{j^{\prime}}(k) m c_{1}^{j^{\prime \prime}}(m)\right) \mid \varrho^{j}(n)\right\rangle \varrho^{j}(n) \\
& +\sum_{1 \leq j, j^{\prime}, j^{\prime \prime} \leq 4} \sum_{k+m=n} e^{-i \frac{t}{\varepsilon} \omega_{k, m, n}^{j, j^{\prime}, j^{\prime \prime}}}\left\langle\left(v_{2}^{j^{\prime}}(k) m c_{2}^{j^{\prime \prime}}(m)\right) \mid \varrho^{j}(n)\right\rangle \varrho^{j}(n) \\
& -\sum_{1 \leq j, j^{\prime}, j^{\prime \prime} \leq 4} \sum_{k+m=n} e^{-i \frac{t}{\varepsilon} \omega_{k, m, n}^{j, j^{\prime}, j^{\prime \prime}}}\left\langle\left(c_{1}^{j^{\prime}}(k) m v_{1}^{j^{\prime \prime}}(m)\right) \mid \varrho^{j}(n)\right\rangle \varrho^{j}(n) \\
& -\sum_{1 \leq j, j^{\prime}, j^{\prime \prime} \leq 4} \sum_{k+m=n} e^{-i \frac{t}{\varepsilon} \omega_{k, m, n}^{j, j^{\prime}, j^{\prime \prime}}}\left\langle\left(c_{2}^{j^{\prime}}(k) m v_{2}^{j^{\prime \prime}}(m)\right) \mid \varrho^{j}(n)\right\rangle \varrho^{j}(n) .
\end{aligned}
$$

In the expression above, we have denoted

$$
\begin{gathered}
\omega_{k, m, n}^{j, j^{\prime}, j^{\prime \prime}}:=\omega^{j^{\prime}}(k)+\omega^{j^{\prime \prime}}(m)-\omega^{j}(n), \\
P(n):=\operatorname{Id}-\frac{1}{n_{1}^{2}+n_{2}^{2}+n_{3}^{2}}\left(\begin{array}{ccc}
n_{1}^{2} & n_{1} n_{2} & n_{1} n_{3} \\
n_{2} n_{1} & n_{2}^{2} & n_{2} n_{3} \\
n_{3} n_{1} & n_{3} n_{2} & n_{3}^{2}
\end{array}\right)
\end{gathered}
$$

and for any vector $X$,

$$
x^{j}:=\left\langle\mathcal{F}(X)(n) \mid \varrho^{j}(n)\right\rangle \varrho^{j}(n) .
$$

The filtering procedure presents the advantage that the singular perturbation disappears. So, contrarily to $\partial_{t} U^{\varepsilon}$ in system $\left(\mathcal{S}^{\varepsilon}\right), \partial_{t} V^{\varepsilon}$ in system $\left(\tilde{\mathcal{S}}^{\varepsilon}\right)$ is bonded in $L^{\infty}\left([0, T], H^{-N}\left(\mathbb{T}^{3}\right)\right)$ for some sufficiently large integer $N$. Consequently, classical compactness argument leads to $U^{\varepsilon} \rightarrow U$ in $C\left([0, T], H^{s, s^{\prime}}\left(\mathbb{T}^{3}\right)\right)$ for all $s<0$ and $s^{\prime}<\frac{1}{2}$.

As in [8], one uses the stationary phase theorem to show that, in $\mathcal{D}^{\prime}$,

$$
\lim _{\varepsilon \rightarrow 0} Q^{\varepsilon}\left(V^{\varepsilon}, V^{\varepsilon}\right)=Q^{0}(V, V) .
$$

Here $Q^{0}$ is defined by

$$
\mathcal{F}\left(Q^{0}(V, V)\right)(n)=\left(\begin{array}{l}
a_{11}^{0} \\
a_{21}^{0}
\end{array}\right)
$$

where

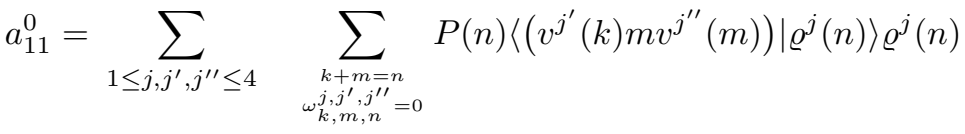

$$
\begin{aligned}
& -\sum_{1 \leq j, j^{\prime}, j^{\prime \prime} \leq 4} \sum_{\substack{k+m=n \\
\omega_{j, j}^{\prime, j}, j^{\prime \prime} \\
k, m, n}} P(n)\left\langle\left(c^{j^{\prime}}(k) m c^{j^{\prime \prime}}(m)\right) \mid \varrho^{j}(n)\right\rangle \varrho^{j}(n)
\end{aligned}
$$


and

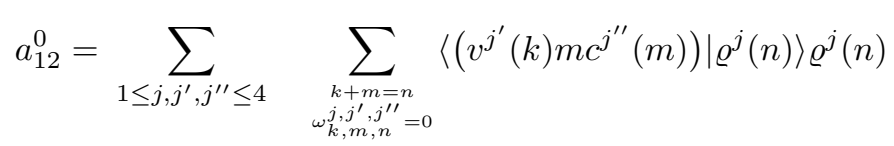

$$
\begin{aligned}
& -\sum_{1 \leq j, j^{\prime}, j^{\prime \prime} \leq 4} \sum_{\substack{k+m=n \\
j, j^{\prime}, j^{\prime \prime} \\
k, m, n}}\left\langle\left(c^{j^{\prime}}(k) m v^{j^{\prime \prime}}(m)\right) \mid \varrho^{j}(n)\right\rangle \varrho^{j}(n) .
\end{aligned}
$$

When $\varepsilon$ goes to 0 , one obtains formally the following limit system:

$$
(L S)\left\{\begin{array}{l}
\partial_{t} V+Q^{0}(V, V)+a_{2}(D) V=0 \text { in }[0, T] \times \mathbb{T}^{3} \\
\operatorname{div} v=0 \text { in }[0, T] \times \mathbb{T}^{3} \\
\operatorname{div} c=0 \text { in }[0, T] \times \mathbb{T}^{3} \\
\left.V\right|_{t=0}=U_{0}=\left(u_{0}, b_{0}\right) \text { in } \mathbb{T}^{3}
\end{array}\right.
$$

The study of $(L S)$ is given in the following theorem:

Theorem 3. Let $s>\frac{1}{2}$ be a real number and $U_{0}=\left(u_{0}, b_{0}\right) \in H^{0, s}\left(\mathbb{T}^{3}\right)$ be a pair of divergence-free vector fields. Then, there exist a time $T>0$ and $a$ unique solution $V=(v, c)$ of $(L S)$ satisfying

$$
V \in L_{T}^{\infty}\left(H^{0, s}\left(\mathbb{T}^{3}\right)\right) \cap L_{T}^{2}\left(H^{1, s}\left(\mathbb{T}^{3}\right)\right) .
$$

Moreover, $V$ satisfies the following energy estimate

$$
\begin{aligned}
\|V(t, .)\|_{H^{0, s}\left(\mathbb{T}^{3}\right)}^{2} & +\nu \int_{0}^{t}\left\|\nabla_{h} v(\tau, .)\right\|_{H^{0, s}\left(\mathbb{T}^{3}\right)}^{2} d \tau \\
& +\eta \int_{0}^{t}\left\|\nabla_{h} c(\tau, .)\right\|_{H^{0, s}\left(\mathbb{T}^{3}\right)}^{2} d \tau \leq\left\|U_{0}\right\|_{H^{0, s}\left(\mathbb{T}^{3}\right)}^{2} .
\end{aligned}
$$

Furthermore, there exists a constant c such that if

$$
\left\|U_{0}\right\|_{H^{0, s}\left(\mathbb{T}^{3}\right)} \leq c \min (\nu, \eta),
$$

then the solution is global.

The proof follows the lines of the one of Theorem 1 .

Remark 1 . Note that the time $T$ appearing in Theorem 3 can be different from the one of Theorem 1. Note, also, that we are not dealing, here, with the possible relation between the life span of the solution of the system $\left(\mathcal{S}^{\varepsilon}\right)$ and the life span of the solution of the corresponding limit system $(L S)$. But since we are proving a local in time convergence result, any uniform time of existence of both systems, denoted also $T$, is suitable for our purpose.

\subsection{Proof of Theorem 2}

Let

$$
W^{\varepsilon}=V^{\varepsilon}-V=\left(v^{\varepsilon}-v, c^{\varepsilon}-c\right)=\left(W_{1}^{\varepsilon}, W_{2}^{\varepsilon}\right)
$$


$W^{\varepsilon}$ satisfies

$$
\left\{\begin{array}{l}
\partial_{t} W^{\varepsilon}+Q^{\varepsilon}\left(W^{\varepsilon}, W^{\varepsilon}+2 V\right)+a_{2}(D) W^{\varepsilon}=R_{o s c}^{\varepsilon} \text { in }[0, T] \times \mathbb{T}^{3} \\
\operatorname{div} W_{1}^{\varepsilon}=0 \text { in }[0, T] \times \mathbb{T}^{3} \\
\operatorname{div} W_{2}^{\varepsilon}=0 \text { in }[0, T] \times \mathbb{T}^{3} \\
\left.W^{\varepsilon}\right|_{t=0}=(0,0) \text { in } \mathbb{T}^{3}
\end{array}\right.
$$

where

$$
R_{o s c}^{\varepsilon}=Q^{0}(V, V)-Q^{\varepsilon}(V, V)
$$

that is the part of $Q^{\varepsilon}(V, V)$ fulfilling the condition $\omega_{k, m, n}^{j, j^{\prime}, j^{\prime \prime}} \neq 0$. In Fourier variables, we have

$$
\mathcal{F}\left(R_{\text {osc }}^{\varepsilon}\right)(n)=\left(\begin{array}{l}
a_{11 \text { osc }} \\
a_{21 \text { osc }}
\end{array}\right)
$$

where

$$
\begin{aligned}
a_{11 o s c}= & \sum_{1 \leq j, j^{\prime}, j^{\prime \prime} \leq 4} \sum_{\substack{k+m=n \\
\omega_{k, j^{\prime}, j^{\prime \prime}} \neq 0}} e^{-i \frac{t}{\varepsilon} \omega_{k, m, n}^{j, j^{\prime}, j^{\prime \prime}}} P(n)\left\langle\left(v^{j^{\prime}}(k) m v^{j^{\prime \prime}}(m)\right) \mid \varrho^{j}(n)\right\rangle \varrho^{j}(n) \\
& -\sum_{1 \leq j, j^{\prime}, j^{\prime \prime} \leq 4} \sum_{\substack{k+m=n \\
j, j^{\prime}, j^{\prime \prime} \\
k, m, n}} e^{-i \frac{t}{\varepsilon} \omega_{k, m, n}^{j, j^{\prime}, j^{\prime \prime}}} P(n)\left\langle\left(c^{j^{\prime}}(k) m c^{j^{\prime \prime}}(m)\right) \mid \varrho^{j}(n)\right\rangle \varrho^{j}(n)
\end{aligned}
$$

and

$$
\begin{aligned}
a_{21 \text { osc }}= & \sum_{1 \leq j, j^{\prime}, j^{\prime \prime} \leq 4} \sum_{\substack{k+m=n \\
\omega_{k, j^{\prime}, j^{\prime \prime} \neq 0} \neq 0}} e^{-i \frac{t}{\varepsilon} \omega_{k, m, n}^{j, j^{\prime}, j^{\prime \prime}}}\left\langle\left(v^{j^{\prime}}(k) m c^{j^{\prime \prime}}(m)\right) \mid \varrho^{j}(n)\right\rangle \varrho^{j}(n) \\
& -\sum_{1 \leq j, j^{\prime}, j^{\prime \prime} \leq 4} \sum_{\substack{k+m=n \\
\omega_{k, j^{\prime}, j^{\prime \prime} \neq 0} \neq m, n}} e^{-i \frac{t}{\varepsilon} \omega_{k, m, n}^{j, j^{\prime}, j^{\prime \prime}}}\left\langle\left(c^{j^{\prime}}(k) m v^{j^{\prime \prime}}(m)\right) \mid \varrho^{j}(n)\right\rangle \varrho^{j}(n) .
\end{aligned}
$$

The right hand side of system (10) is an oscillating term which converges weakly to zero but not strongly. The method we use here to deal with $R_{o s c}^{\varepsilon}$ is inspired from the ideas introduced by $\mathrm{S}$. Schochet in [13]. It consists in dividing $R_{o s c}^{\varepsilon}$ into high frequency term $R_{o s c}^{\varepsilon, N}$ and low frequency term $R_{o s c, N}^{\varepsilon}$ defined respectively, for any arbitrary cut-off integer $N \geq 1$, by

$$
R_{o s c}^{\varepsilon, N}=R_{o s c}^{\varepsilon}-R_{o s c, N}^{\varepsilon}
$$

and

$$
\mathcal{F}\left(R_{o s c, N}^{\varepsilon}\right)(t, n)=1_{\{|n| \leq N\}} \mathcal{F}\left(R_{o s c ;|k|,|m| \leq N}^{\varepsilon}\right)(t, n) .
$$

Here, $R_{o s c ;|k|,|m| \leq N}^{\varepsilon}$ is the part of $R_{o s c}^{\varepsilon}$ for which the variables $k$ and $m$ are such that $|k|,|m| \leq N$. Explicitly, we have

$$
\mathcal{F}\left(R_{o s c, N}^{\varepsilon}\right)(n)=\left(\begin{array}{c}
a_{11 o s c, N} \\
a_{21 o s c, N}
\end{array}\right)
$$


where

$$
\begin{aligned}
& a_{11 \text { osc }, N}
\end{aligned}
$$

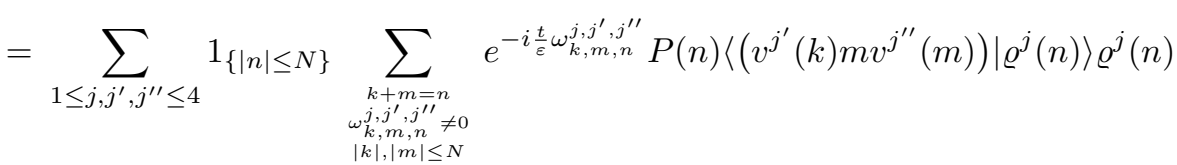

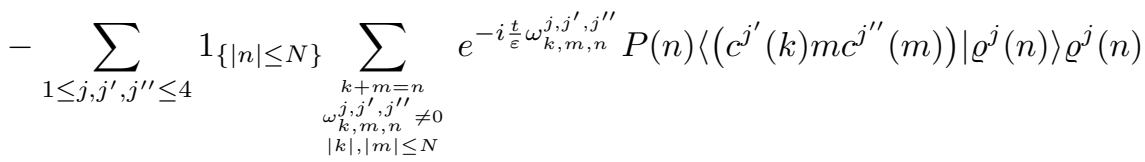

and

$$
\begin{aligned}
& a_{21 \text { osc, } N}
\end{aligned}
$$

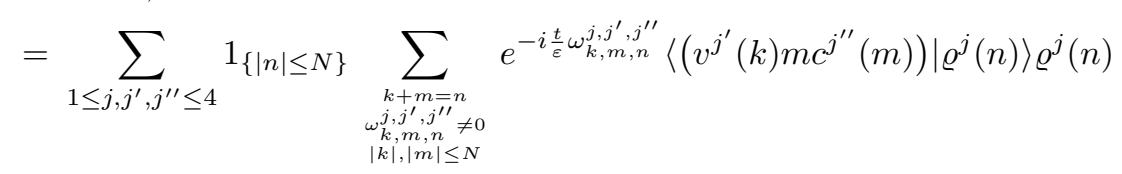

$$
\begin{aligned}
& -\sum_{1 \leq j, j^{\prime}, j^{\prime \prime} \leq 4} 1_{\{|n| \leq N\}} \sum_{\substack{k+m=n \\
\text { a.j, } \\
k, m, j j^{\prime \prime} \neq 0 \\
|k|,|m| \leq N}} e^{-i \frac{t}{\varepsilon} \omega_{k, m, n}^{j, j^{\prime}, j^{\prime \prime}}}\left\langle\left(c^{j^{\prime}}(k) m v^{j^{\prime \prime}}(m)\right) \mid \varrho^{j}(n)\right\rangle \varrho^{j}(n) .
\end{aligned}
$$

To absorb the low frequency term, we adopt the following change of function:

$$
\varphi_{N}^{\varepsilon}=W^{\varepsilon}+\varepsilon \tilde{R}_{o s c, N}^{\varepsilon} .
$$

In term of Fourier variables, $\tilde{R}_{o s c, N}^{\varepsilon}$ is defined by

$$
\mathcal{F}\left(\tilde{R}_{o s c, N}^{\varepsilon}\right)(n)=\left(\begin{array}{c}
\tilde{a}_{11 o s c, N} \\
\tilde{a}_{21 o s c, N}
\end{array}\right),
$$

where

$$
\begin{aligned}
& \tilde{a}_{11 o s c, N}
\end{aligned}
$$

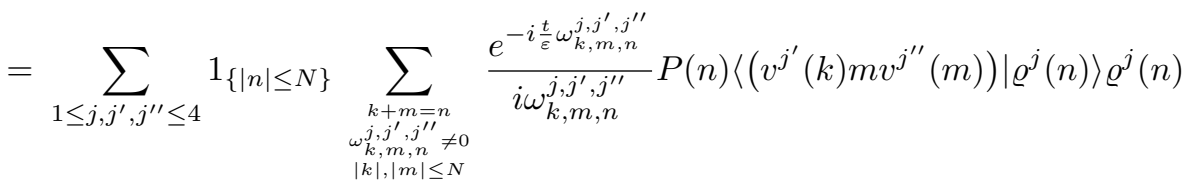

$$
\begin{aligned}
& -\sum_{1 \leq j, j^{\prime}, j^{\prime \prime} \leq 4} 1_{\{|n| \leq N\}} \sum_{\substack{k+m=n \\
\omega_{k, j^{\prime}, j^{\prime \prime}} \neq 0 \\
|k|,|m| \leq N}} \frac{e^{-i \frac{t}{\varepsilon} \omega_{k, m, n}^{j, j^{\prime}, j^{\prime \prime}}}}{i \omega_{k, m, n}^{j, j^{\prime}, j^{\prime \prime}}} P(n)\left\langle\left(c^{j^{\prime}}(k) m c^{j^{\prime \prime}}(m)\right) \mid \varrho^{j}(n)\right\rangle \varrho^{j}(n)
\end{aligned}
$$

and 


$$
\begin{aligned}
& \tilde{a}_{21 o s c, N}
\end{aligned}
$$

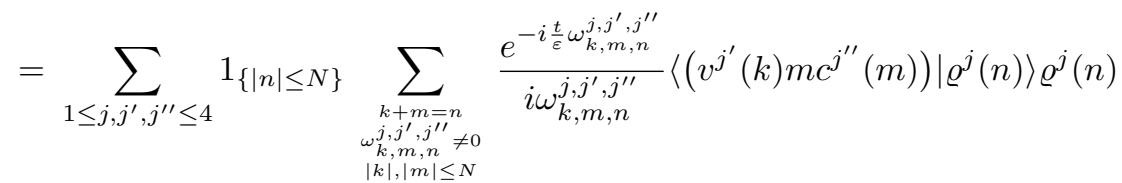

$$
\begin{aligned}
& -\sum_{1 \leq j, j^{\prime}, j^{\prime \prime} \leq 4} 1_{\{|n| \leq N\}} \sum_{\substack{k+m=n \\
\omega^{j, j}, j^{\prime}, n \neq 0 \\
k, m, n \\
k|,| m \mid \leq N}} \frac{e^{-i \frac{t}{\varepsilon} \omega_{k, m, n}^{j, j^{\prime}, j^{\prime \prime}}}}{i \omega_{k, m, n}^{j, j^{\prime}, j^{\prime \prime}}}\left\langle\left(c^{j^{\prime}}(k) m v^{j^{\prime \prime}}(m)\right) \mid \varrho^{j}(n)\right\rangle \varrho^{j}(n) .
\end{aligned}
$$

The new function $\varphi_{N}^{\varepsilon}$ satisfies the following equation:

$$
\partial_{t} \varphi_{N}^{\varepsilon}+Q^{\varepsilon}\left(\varphi_{N}^{\varepsilon}, \varphi_{N}^{\varepsilon}-2 \varepsilon \tilde{R}_{o s c, N}^{\varepsilon}+2 V\right)+a_{2}(D) \varphi_{N}^{\varepsilon}=R_{o s c}^{\varepsilon, N}+\varepsilon r_{o s c, N}^{\varepsilon},
$$

where

$$
\varepsilon r_{o s c, N}^{\varepsilon}=-Q^{\varepsilon}\left(\varepsilon \tilde{R}_{o s c, N}^{\varepsilon}, \varepsilon \tilde{R}_{o s c, N}^{\varepsilon}-2 V\right)-\varepsilon a_{2}(D)\left(\tilde{R}_{o s c, N}^{\varepsilon}\right)+\varepsilon \tilde{R}_{o s c, N}^{\varepsilon, t} .
$$

In Fourier variables, $\tilde{R}_{o s c, N}^{\varepsilon, t}$ is explicitly given by

$$
\mathcal{F}\left(\tilde{R}_{o s c, N}^{\varepsilon, t}\right)(n)=\left(\begin{array}{l}
\tilde{a}_{11 o s c, N}^{t} \\
\tilde{a}_{21 o s c, N}^{t}
\end{array}\right),
$$

where

$$
\begin{aligned}
& \tilde{a}_{11 \text { osc }, N}^{t}
\end{aligned}
$$

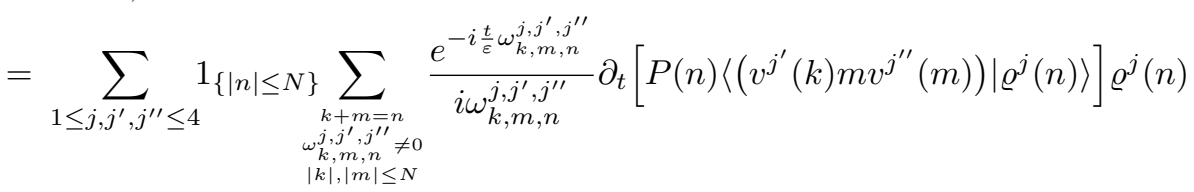

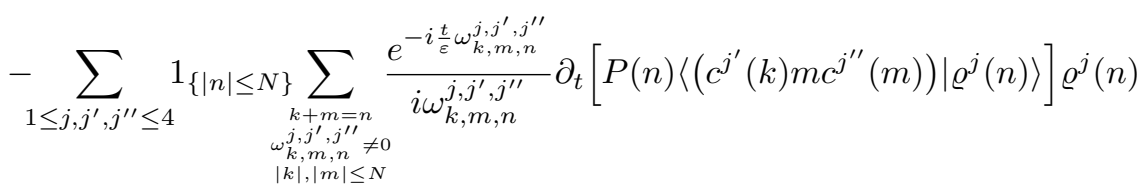

and

$$
\begin{aligned}
& \tilde{a}_{21 \text { osc }, N}^{t} \\
& =\sum_{1 \leq j, j^{\prime}, j^{\prime \prime} \leq 4} 1_{\{|n| \leq N\}} \sum_{\substack{k+m=n \\
\text { wa, } \\
k, j^{\prime}, j^{\prime \prime} \neq 0 \\
|k|,|m| \leq N}} \frac{e^{-i \frac{t}{\varepsilon} \omega_{k, m, n}^{j, j^{\prime}, j^{\prime \prime}}}}{i \omega_{k, m, n}^{j, j^{\prime}, j^{\prime \prime}}} \partial_{t}\left[\left\langle\left(v^{j^{\prime}}(k) m c^{j^{\prime \prime}}(m)\right) \mid \varrho^{j}(n)\right\rangle\right] \varrho^{j}(n) \\
& -\sum_{1 \leq j, j^{\prime}, j^{\prime \prime} \leq 4} 1_{\{|n| \leq N\}} \sum_{\substack{k+m=n \\
\omega_{k, j^{\prime}, j^{\prime \prime} \neq 0} \\
|k|,|m| \leq N}} \frac{e^{-i \frac{t}{\varepsilon} \omega_{k, m, n}^{j, j^{\prime}, j^{\prime \prime}}}}{i \omega_{k, m, n}^{j, j^{\prime}, j^{\prime \prime}}} \partial_{t}\left[\left\langle\left(c^{j^{\prime}}(k) m v^{j^{\prime \prime}}(m)\right) \mid \varrho^{j}(n)\right\rangle\right] \varrho^{j}(n) .
\end{aligned}
$$


We note that the equation satisfied by $\varphi_{N}^{\varepsilon}$ has the advantage that the low frequency terms have disappeared up to an $\varepsilon$. Let us denote for any real number $x$, by $\langle x\rangle$ the quantity $\left(1+|x|^{2}\right)^{\frac{1}{2}}$ and by $\Lambda_{3}$ the operator defined by

$$
\Lambda_{3}=\left(1+\partial_{3}^{2}\right)^{\frac{1}{2}}
$$

that is the operator of multiplication by $\left\langle k_{3}\right\rangle$ in the frequency space. Clearly, for all real numbers $s$ and $s^{\prime}, \Lambda_{3}$ is an isometry from $H^{s, s^{\prime}}$ to $H^{s, s^{\prime}-1}$.

Taking the scalar product in $H^{0,-\frac{1}{2}}\left(\mathbb{T}^{3}\right)$, equation (12) gives

$$
\begin{aligned}
& \left\|\varphi_{N}^{\varepsilon}\right\|_{0,-\frac{1}{2}}^{2}+2 \nu \int_{0}^{t}\left\|\nabla_{h} \varphi_{N, 1}^{\varepsilon}\right\|_{0,-\frac{1}{2}}^{2} d \tau+2 \eta \int_{0}^{t}\left\|\nabla_{h} \varphi_{N, 2}^{\varepsilon}\right\|_{0,-\frac{1}{2}}^{2} d \tau \\
= & -2 \int_{0}^{t} \int_{\mathbb{T}^{3}} Q^{\varepsilon}\left(\varphi_{N}^{\varepsilon}, \varphi_{N}^{\varepsilon}-2 \varepsilon \tilde{R}_{o s c, N}^{\varepsilon}+2 V\right) \Lambda_{3}^{-1} \varphi_{N}^{\varepsilon} d x d \tau \\
& -2 \int_{0}^{t} \int_{\mathbb{T}^{3}}\left(R_{o s c}^{\varepsilon, N}+\varepsilon r_{o s c, N}^{\varepsilon}\right) \Lambda_{3}^{-1} \varphi_{N}^{\varepsilon} d x d \tau .
\end{aligned}
$$

To estimate the nonlinear part, we note that

$$
\int_{\mathbb{T}^{3}} Q^{\varepsilon}\left(\varphi_{N}^{\varepsilon}, \varphi_{N}^{\varepsilon}-2 \varepsilon \tilde{R}_{o s c, N}^{\varepsilon}+2 V\right) \Lambda_{3}^{-1} \varphi_{N}^{\varepsilon} d x d \tau=\sum_{i=1}^{4} I_{i},
$$

where

$$
\begin{aligned}
& I_{1}=\int_{\mathbb{T}^{3}} \mathcal{L}\left(\frac{-t}{\varepsilon}\right) \mathbb{P}\left[\left(\mathcal{L}\left(\frac{t}{\varepsilon}\right)\left(\varphi_{N}^{\varepsilon}-2 \varepsilon \tilde{R}_{o s c, N}^{\varepsilon}+2 V\right)\right)_{h} \nabla_{h}\left(\mathcal{L}\left(\frac{t}{\varepsilon}\right) \varphi_{N}^{\varepsilon}\right)\right] \Lambda_{3}^{-1} \varphi_{N}^{\varepsilon} d x, \\
& I_{2}=\int_{\mathbb{T}^{3}} \mathcal{L}\left(\frac{-t}{\varepsilon}\right) \mathbb{P}\left[\left(\mathcal{L}\left(\frac{t}{\varepsilon}\right)\left(\varphi_{N}^{\varepsilon}-2 \varepsilon \tilde{R}_{o s c, N}^{\varepsilon}+2 V\right)\right)_{3} \partial_{3}\left(\mathcal{L}\left(\frac{t}{\varepsilon}\right) \varphi_{N}^{\varepsilon}\right)\right] \Lambda_{3}^{-1} \varphi_{N}^{\varepsilon} d x, \\
& I_{3}=\int_{\mathbb{T}^{3}} \mathcal{L}\left(\frac{-t}{\varepsilon}\right) \mathbb{P}\left[\left(\mathcal{L}\left(\frac{t}{\varepsilon}\right) \varphi_{N}^{\varepsilon}\right)_{h} \nabla_{h}\left(\mathcal{L}\left(\frac{t}{\varepsilon}\right)\left(\varphi_{N}^{\varepsilon}-2 \varepsilon \tilde{R}_{o s c, N}^{\varepsilon}+2 V\right)\right)\right] \Lambda_{3}^{-1} \varphi_{N}^{\varepsilon} d x
\end{aligned}
$$

and

$$
I_{4}=\int_{\mathbb{T}^{3}} \mathcal{L}\left(\frac{-t}{\varepsilon}\right) \mathbb{P}\left[\left(\mathcal{L}\left(\frac{t}{\varepsilon}\right) \varphi_{N}^{\varepsilon}\right)_{3} \partial_{3}\left(\mathcal{L}\left(\frac{t}{\varepsilon}\right)\left(\varphi_{N}^{\varepsilon}-2 \varepsilon \tilde{R}_{o s c, N}^{\varepsilon}+2 V\right)\right)\right] \Lambda_{3}^{-1} \varphi_{N}^{\varepsilon} d x
$$

To estimate $I_{1}$, one uses that $\mathcal{L}$ is an isometry and $\mathbb{P}$ is a projection to obtain, by duality, that

$$
\left|I_{1}\right| \leq\left\|\mathcal{L}\left(\frac{t}{\varepsilon}\right)\left(\varphi_{N}^{\varepsilon}-2 \varepsilon \tilde{R}_{o s c, N}^{\varepsilon}+2 V\right)_{h} \nabla_{h}\left(\mathcal{L}\left(\frac{t}{\varepsilon}\right) \varphi_{N}^{\varepsilon}\right)\right\|_{-\frac{1}{2},-\frac{1}{2}}\left\|\Lambda_{3}^{-1} \varphi_{N}^{\varepsilon}\right\|_{\frac{1}{2}, \frac{1}{2}} .
$$

Before going any farther, we recall the following anisotropic law stated in [10].

Lemma 2. Let $s, t<1, s+t>0$ and $s^{\prime}>\frac{1}{2}$. If $f$ belongs to $H^{s, s^{\prime}}$ and $g$ belongs to $H^{s,-\frac{1}{2}}$, then $f g$ belongs to $H^{s+t-1,-\frac{1}{2}}$ and there exists a constant $C$ such that

$$
\|f g\|_{H^{s+t-1,-\frac{1}{2}}} \leq C\|f\|_{H^{s, s^{\prime}}}\|g\|_{H^{t,-\frac{1}{2}}} .
$$


Its originality, as said in [10], is to give a product law in anisotropic Sobolev spaces, where the regularities in the vertical direction are supercritical for one of the terms and subcritical for the other. Then using Lemma 2, and the fact that $\mathcal{L}$ and $\Lambda_{3}$ are both isometries, one deduces that

$$
\left|I_{1}\right| \leq\left\|\varphi_{N}^{\varepsilon}-2 \varepsilon \tilde{R}_{o s c, N}^{\varepsilon}+2 V\right\|_{\frac{1}{2}, s}\left\|\varphi_{N}^{\varepsilon}\right\|_{1,-\frac{1}{2}}\left\|\varphi_{N}^{\varepsilon}\right\|_{\frac{1}{2},-\frac{1}{2}} .
$$

Finally, interpolation inequality implies that

$$
\left|I_{1}\right| \leq\left\|\varphi_{N}^{\varepsilon}-2 \varepsilon \tilde{R}_{o s c, N}^{\varepsilon}+2 V\right\|_{\frac{1}{2}, s}\left\|\varphi_{N}^{\varepsilon}\right\|_{0,-\frac{1}{2}}^{\frac{1}{2}}\left\|\varphi_{N}^{\varepsilon}\right\|_{1,-\frac{1}{2}}^{\frac{3}{2}} .
$$

The same holds for $I_{3}$, and one has

$$
\begin{aligned}
\left|I_{3}\right| & \leq\left\|\left(\mathcal{L}\left(\frac{t}{\varepsilon}\right) \varphi_{N}^{\varepsilon}\right)_{h} \nabla_{h}\left(\mathcal{L}\left(\frac{t}{\varepsilon}\right)\left(\varphi_{N}^{\varepsilon}-2 \varepsilon \tilde{R}_{o s c, N}^{\varepsilon}+2 V\right)\right)\right\|_{-\frac{3}{4},-\frac{1}{2}}\left\|\Lambda_{3}^{-1} \varphi_{N}^{\varepsilon}\right\|_{\frac{3}{4}, \frac{1}{2}} \\
& \leq C\left\|\left(\varphi_{N}^{\varepsilon}-2 \varepsilon \tilde{R}_{o s c, N}^{\varepsilon}+2 V\right)\right\|_{\frac{1}{2}, s}\left\|\varphi_{N}^{\varepsilon}\right\|_{\frac{3}{4},-\frac{1}{2}}^{2} .
\end{aligned}
$$

This leads to

$$
\left|I_{3}\right| \leq C\left\|\left(\varphi_{N}^{\varepsilon}-2 \varepsilon \tilde{R}_{o s c, N}^{\varepsilon}+2 V\right)\right\|_{\frac{1}{2}, s}\left\|\varphi_{N}^{\varepsilon}\right\|_{0,-\frac{1}{2}}^{\frac{1}{2}}\left\|\varphi_{N}^{\varepsilon}\right\|_{1,-\frac{1}{2}}^{\frac{3}{2}} .
$$

To estimate $I_{4}$, one has by duality that

$$
\left|I_{4}\right| \leq\left\|\left(\mathcal{L}\left(\frac{t}{\varepsilon}\right) \varphi_{N}^{\varepsilon}\right)_{3} \partial_{3}\left(\mathcal{L}\left(\frac{t}{\varepsilon}\right)\left(\varphi_{N}^{\varepsilon}-2 \varepsilon \tilde{R}_{o s c, N}^{\varepsilon}+2 V\right)\right)\right\|_{-\frac{1}{2}, \frac{2 s-3}{4}}\left\|\Lambda_{3}^{-1} \varphi_{N}^{\varepsilon}\right\|_{\frac{1}{2}, \frac{3-2 s}{4}} .
$$

To continue investigating $I_{4}$, we recall the following product law proved in [9]:

Lemma 3. Let $s, t<1, s+t>0$ and $s^{\prime}, t^{\prime}<\frac{1}{2}, s^{\prime}+t^{\prime}>0$. If $f$ belongs to $H^{s, s^{\prime}}$ and $g$ belongs to $H^{t, t^{\prime}}$, then fg belongs to $H^{s+t-1, s^{\prime}+t^{\prime}-\frac{1}{2}}$ and there exists a constant $C$ such that

$$
\|f g\|_{H^{s+t-1, s^{\prime}+t^{\prime}-\frac{1}{2}}} \leq C\|f\|_{H^{s, s^{\prime}}}\|g\|_{H^{t, t^{\prime}}} .
$$

This lemma implies that

$$
\left|I_{4}\right| \leq C\left\|\left(\varphi_{N}^{\varepsilon}\right)_{3}\right\|_{0, \frac{3-2 s}{4}}\left\|\partial_{3}\left(\varphi_{N}^{\varepsilon}-2 \varepsilon \tilde{R}_{o s c, N}^{\varepsilon}+2 V\right)\right\|_{\frac{1}{2}, s-1}\left\|\varphi_{N}^{\varepsilon}\right\|_{\frac{1}{2}, \frac{-2 s-1}{4}} .
$$

Without loss of generality, we can assume that $s<1$, to obtain

$$
\left|I_{4}\right| \leq C\left\|\left(\varphi_{N}^{\varepsilon}\right)_{3}\right\|_{0, \frac{1}{2}}\left\|\varphi_{N}^{\varepsilon}-2 \varepsilon \tilde{R}_{o s c, N}^{\varepsilon}+2 V\right\|_{\frac{1}{2}, s}\left\|\varphi_{N}^{\varepsilon}\right\|_{\frac{1}{2},-\frac{1}{2}} .
$$

However, since we are working in the framework of divergence free condition, $\varphi_{N}^{\varepsilon}$ is also a divergence free vector satisfying

$$
\begin{aligned}
\left\|\left(\varphi_{N}^{\varepsilon}\right)_{3}\right\|_{0, \frac{1}{2}} & \leq\left\|\varphi_{N}^{\varepsilon}\right\|_{0,-\frac{1}{2}}+\left\|\partial_{3} \varphi_{N, 3}^{\varepsilon}\right\|_{0,-\frac{1}{2}} \\
& \leq\left\|\varphi_{N}^{\varepsilon}\right\|_{0,-\frac{1}{2}}+\left\|\operatorname{div}_{h} \varphi_{N, h}^{\varepsilon}\right\|_{0,-\frac{1}{2}} \\
& \leq\left\|\varphi_{N}^{\varepsilon}\right\|_{0,-\frac{1}{2}}+\left\|\varphi_{N}^{\varepsilon}\right\|_{1,-\frac{1}{2}} .
\end{aligned}
$$

By interpolation, we infer that

$$
\left|I_{4}\right| \leq C\left\|\varphi_{N}^{\varepsilon}-2 \varepsilon \tilde{R}_{o s c, N}^{\varepsilon}+2 V\right\|_{\frac{1}{2}, s}\left(\left\|\varphi_{N}^{\varepsilon}\right\|_{0,-\frac{1}{2}}^{\frac{3}{2}}\left\|\varphi_{N}^{\varepsilon}\right\|_{1,-\frac{1}{2}}^{\frac{1}{2}}+\left\|\varphi_{N}^{\varepsilon}\right\|_{0,-\frac{1}{2}}^{\frac{1}{2}}\left\|\varphi_{N}^{\varepsilon}\right\|_{1,-\frac{1}{2}}^{\frac{3}{2}}\right) .
$$


To estimate $I_{2}$, we note that

$$
I_{2}=\int_{\mathbb{T}^{3}} \mathcal{L}\left(\frac{-t}{\varepsilon}\right) \mathbb{P}\left[\left(\mathcal{L}\left(\frac{t}{\varepsilon}\right)\left(\varphi_{N}^{\varepsilon}-2 \varepsilon \tilde{R}_{o s c, N}^{\varepsilon}+2 V\right)\right)_{3} \partial_{3}\left(\mathcal{L}\left(\frac{t}{\varepsilon}\right) \varphi_{N}^{\varepsilon}\right)\right] \Lambda_{3}^{-1} \varphi_{N}^{\varepsilon} .
$$

Using Parseval's formula, we have

$$
\begin{array}{rl}
I_{2}=(2 \pi)^{-3} \sum_{k \in \mathbb{Z}} & \mathcal{F}\left(\mathcal{L}\left(\frac{-t}{\varepsilon}\right) \mathbb{P}\left[\left(\mathcal{L}\left(\frac{t}{\varepsilon}\right)\left(\varphi_{N}^{\varepsilon}-2 \varepsilon \tilde{R}_{o s c, N}^{\varepsilon}+2 V\right)\right)_{3} \partial_{3}\left(\mathcal{L}\left(\frac{t}{\varepsilon}\right) \varphi_{N}^{\varepsilon}\right)\right]\right)(k) \\
& \times \mathcal{F}\left(\Lambda_{3}^{-1} \varphi_{N}^{\varepsilon}\right)(-k) .
\end{array}
$$

According to the definition of $\Lambda_{3}$, we have

$$
\begin{array}{rl}
I_{2}=(2 \pi)^{-3} \sum_{k \in \mathbb{Z}} & \mathcal{F}\left(\mathcal{L}\left(\frac{-t}{\varepsilon}\right) \mathbb{P}\left[\left(\mathcal{L}\left(\frac{t}{\varepsilon}\right)\left(\varphi_{N}^{\varepsilon}-2 \varepsilon \tilde{R}_{o s c, N}^{\varepsilon}+2 V\right)\right)_{3} \partial_{3}\left(\mathcal{L}\left(\frac{t}{\varepsilon}\right) \varphi_{N}^{\varepsilon}\right)\right]\right)(k) \\
& \times \frac{1}{\left\langle k_{3}\right\rangle} \mathcal{F}\left(\varphi_{N}^{\varepsilon}\right)(-k) .
\end{array}
$$

Using the following property of $\mathbb{P}$ :

$$
\mathcal{F}(\mathbb{P} u)=\mathcal{F}(u)-\left\langle\mathcal{F}(u), \frac{k}{|k|}\right\rangle \frac{k}{|k|},
$$

we can rewrite $I_{2}$ in the following form:

$$
\begin{gathered}
I_{2}=(2 \pi)^{-3} \sum_{k \in \mathbb{Z}}\left\{\mathcal{F}\left(\mathcal{L}\left(\frac{-t}{\varepsilon}\right)\left[\left(\mathcal{L}\left(\frac{t}{\varepsilon}\right)\left(\varphi_{N}^{\varepsilon}-2 \varepsilon \tilde{R}_{\text {osc }, N}^{\varepsilon}+2 V\right)\right)_{3} \partial_{3}\left(\mathcal{L}\left(\frac{t}{\varepsilon}\right) \varphi_{N}^{\varepsilon}\right)\right]\right)(k)\right. \\
-\left\langle\mathcal{F}\left(\mathcal{L}\left(\frac{-t}{\varepsilon}\right)\left[\left(\mathcal{L}\left(\frac{t}{\varepsilon}\right)\left(\varphi_{N}^{\varepsilon}-2 \varepsilon \tilde{R}_{o s c, N}^{\varepsilon}+2 V\right)\right)_{3} \partial_{3}\left(\mathcal{L}\left(\frac{t}{\varepsilon}\right) \varphi_{N}^{\varepsilon}\right)\right]\right)(k),\right. \\
\left.\left.\frac{k}{|k|}\right\rangle \frac{k}{|k|}\right\} \frac{1}{\left\langle k_{3}\right\rangle} \mathcal{F}\left(\varphi_{N}^{\varepsilon}\right)(-k) .
\end{gathered}
$$

Since

$$
\mathcal{L}\left(\frac{t}{\varepsilon}\right)(U)(t, k)=\mathcal{F}^{-1}\left(\sum_{j \in\{1,2,3,4\}} \exp \left(\omega_{j}(k) \frac{t}{\varepsilon}\right)\left\langle\mathcal{F}(U)(k), \varrho^{j}(k)\right\rangle \varrho^{j}(k)\right),
$$

we can deduce that

$$
\begin{aligned}
& \mathcal{F}\left(\mathcal{L}\left(\frac{-t}{\varepsilon}\right)\left[\left(\mathcal{L}\left(\frac{t}{\varepsilon}\right)\left(\varphi_{N}^{\varepsilon}-2 \varepsilon \tilde{R}_{o s c, N}^{\varepsilon}+2 V\right)\right)_{3} \partial_{3}\left(\mathcal{L}\left(\frac{t}{\varepsilon}\right) \varphi_{N}^{\varepsilon}\right)\right]\right)(k) \\
= & \sum_{n \in \mathbb{Z}} \sum_{j, j^{\prime}, j^{\prime \prime}} n_{3} \exp \left(\omega_{k-n, n, k}^{j, j^{\prime}, j^{\prime \prime}} \frac{t}{\varepsilon}\right)\left\langle\left(\varphi_{N}^{\varepsilon}-2 \varepsilon \tilde{R}_{o s c, N}^{\varepsilon}+2 V\right)^{j^{\prime}}(k-n)\left(\varphi_{N}^{\varepsilon}\right)^{j^{\prime \prime}}(n) \mid \varrho^{j}(k)\right\rangle \varrho^{j}(k) .
\end{aligned}
$$

Using the change of variable $(k, n) \leftrightarrow(-n,-k)$, we obtain

$$
\begin{aligned}
I_{2}=C \sum_{j, j^{\prime}, j^{\prime \prime}} & \sum_{n \in \mathbb{Z}} \sum_{k \in \mathbb{Z}} \exp \left(\omega_{k-n, n, k}^{j, j^{\prime}, j^{\prime \prime}} \frac{t}{\varepsilon}\right)\left(\frac{n_{3}}{\left\langle k_{3}\right\rangle}-\frac{k_{3}}{\left\langle n_{3}\right\rangle}\right) \times \mathcal{F}\left(\varphi_{N}^{\varepsilon}\right)(-k) \\
& \left\{\left\langle\left(\varphi_{N}^{\varepsilon}-2 \varepsilon \tilde{R}_{o s c, N}^{\varepsilon}+2 V\right)^{j^{\prime}}(k-n)\left(\varphi_{N}^{\varepsilon}\right)^{j^{\prime \prime}}(n) \mid \varrho^{j}(k)\right\rangle \varrho^{j}(k)-\right. \\
& \left.\left\langle\left\langle\left(\varphi_{N}^{\varepsilon}-2 \varepsilon \tilde{R}_{o s c, N}^{\varepsilon}+2 V\right)^{j^{\prime}}(k-n)\left(\varphi_{N}^{\varepsilon}\right)^{j^{\prime \prime}}(n) \mid \varrho^{j}(k)\right\rangle \varrho^{j}(k), \frac{k}{|k|}\right\rangle \frac{k}{|k|}\right\} .
\end{aligned}
$$


We recall the following elementary equality, due to [10]:

$$
\frac{n_{3}}{\left\langle k_{3}\right\rangle}-\frac{k_{3}}{\left\langle n_{3}\right\rangle}=\frac{n_{3}-k_{3}}{\left\langle k_{3}\right\rangle}+\frac{\left(n_{3}-k_{3}\right) k_{3}\left(n_{3}+k_{3}\right)}{\left\langle n_{3}\right\rangle\left\langle k_{3}\right\rangle\left(\left\langle n_{3}\right\rangle+\left\langle k_{3}\right\rangle\right)} .
$$

It implies that

$$
\left|\frac{n_{3}}{\left\langle k_{3}\right\rangle}-\frac{k_{3}}{\left\langle n_{3}\right\rangle}\right| \leq\left|n_{3}-k_{3}\right|\left(\frac{1}{\left\langle n_{3}\right\rangle}+\frac{1}{\left\langle k_{3}\right\rangle}\right) .
$$

Then, it follows that

$$
\begin{aligned}
\left|I_{2}\right| \leq C \sum_{n \in \mathbb{Z}} \sum_{k \in \mathbb{Z}} & \left|n_{3}-k_{3}\right|\left(\frac{1}{\left\langle n_{3}\right\rangle}+\frac{1}{\left\langle k_{3}\right\rangle}\right)\left|\mathcal{F}\left(\left(\varphi_{N}^{\varepsilon}-2 \varepsilon \tilde{R}_{o s c, N}^{\varepsilon}+2 V\right)_{3}\right)(k-n)\right| \\
& \times\left|\mathcal{F}\left(\varphi_{N}^{\varepsilon}\right)(n)\right|\left|\mathcal{F}\left(\varphi_{N}^{\varepsilon}\right)(-k)\right| .
\end{aligned}
$$

Using again the change of variables $(k, n) \leftrightarrow(-n,-k)$, we obtain

$\left|I_{2}\right| \leq C \sum_{n \in \mathbb{Z}} \sum_{k \in \mathbb{Z}} \frac{\left|n_{3}-k_{3}\right|}{\left\langle k_{3}\right\rangle}\left|\mathcal{F}\left(\left(\varphi_{N}^{\varepsilon}-2 \varepsilon \tilde{R}_{o s c, N}^{\varepsilon}+2 V\right)_{3}\right)(k-n) \| \mathcal{F}\left(\varphi_{N}^{\varepsilon}\right)(n)\right|\left|\mathcal{F}\left(\varphi_{N}^{\varepsilon}\right)(-k)\right|$.

As $W, V$ and $\varphi_{N}^{\varepsilon}$ are divergence free, we infer that

$$
\begin{aligned}
\left|I_{2}\right| \leq C \sum_{n \in \mathbb{Z}} \sum_{k \in \mathbb{Z}}( & \frac{\left|n_{1}-k_{1}\right|\left|\mathcal{F}\left(\left(\varphi_{N}^{\varepsilon}-2 \varepsilon \tilde{R}_{o s c, N}^{\varepsilon}+2 V\right)_{1}\right)\right|}{\left\langle k_{3}\right\rangle} \\
& \left.+\frac{\left|n_{2}-k_{2}\right|\left|\mathcal{F}\left(\left(\varphi_{N}^{\varepsilon}-2 \varepsilon \tilde{R}_{o s c, N}^{\varepsilon}+2 V\right)_{2}\right)\right|}{\left\langle k_{3}\right\rangle}\right) \\
& \times\left|\mathcal{F}\left(\varphi_{N}^{\varepsilon}\right)(n)\right|\left|\mathcal{F}\left(\varphi_{N}^{\varepsilon}\right)(-k)\right| .
\end{aligned}
$$

Let $V^{\prime}$ be the vector fields whose components satisfy

$$
\mathcal{F}\left(V_{j}^{\prime}\right)=\left|\mathcal{F}\left(V_{j}\right)\right|
$$

and so on. It is clear that for any real number $s$ and $s^{\prime}$ we have

$$
\left\|V_{j}^{\prime}\right\|_{s, s^{\prime}}=\left\|V_{j}\right\|_{s, s^{\prime}} .
$$

Denote $\left|D_{j}\right|$ the operator of multiplication by $\left|\xi_{j}\right|$ and using Parseval's formula we deduce that

$\left|I_{2}\right| \leq C \sum_{k \in \mathbb{Z}}\left(\left|D_{1}\right|\left(\varphi_{N}^{\varepsilon}-2 \varepsilon \tilde{R}_{o s c, N}^{\varepsilon}+2 V\right)_{1}^{\prime}+\left|D_{2}\right|\left(\varphi_{N}^{\varepsilon}-2 \varepsilon \tilde{R}_{o s c, N}^{\varepsilon}+2 V\right)_{2}^{\prime}\right) \varphi_{N}^{\prime \varepsilon} \Lambda_{3} \varphi_{N}^{\prime \varepsilon}$.

Since $\left|D_{j}\right| V_{j}^{\prime}$ and $\partial_{j} V_{j}^{\prime}$ has the same $H^{s, s^{\prime}}$ norm, then as for $I_{3}$

$$
\left|I_{2}\right| \leq C\left\|\varphi_{N}^{\varepsilon}-2 \varepsilon \tilde{R}_{o s c, N}^{\varepsilon}+2 V\right\|_{\frac{1}{2}, s}\left\|\varphi_{N}^{\varepsilon}\right\|_{0,-\frac{1}{2}}^{\frac{1}{2}}\left\|\varphi_{N}^{\varepsilon}\right\|_{1,-\frac{1}{2}}^{\frac{3}{2}} .
$$


Using estimations (15), (16), (17) and (19), equation (14) becomes

$$
\begin{aligned}
& \left\|\varphi_{N}^{\varepsilon}\right\|_{0,-\frac{1}{2}}^{2}+2 \nu \int_{0}^{t}\left\|\nabla_{h} \varphi_{N, 1}^{\varepsilon}\right\|_{0,-\frac{1}{2}}^{2} d \tau+2 \eta \int_{0}^{t}\left\|\nabla_{h} \varphi_{N, 2}^{\varepsilon}\right\|_{0,-\frac{1}{2}}^{2} d \tau \\
\leq & C \int_{0}^{t}\left\|\varphi_{N}^{\varepsilon}\right\|_{1,-\frac{1}{2}}^{\frac{3}{2}}\left\|\varphi_{N}^{\varepsilon}\right\|_{0,-\frac{1}{2}}^{\frac{1}{2}}\left\|\varphi_{N}^{\varepsilon}-2 \varepsilon \tilde{R}_{o s c, N}^{\varepsilon}+2 V\right\|_{\frac{1}{2}, s} d \tau \\
& +C \int_{0}^{t}\left\|\varphi_{N}^{\varepsilon}\right\|_{1,-\frac{1}{2}}^{\frac{1}{2}}\left\|\varphi_{N}^{\varepsilon}\right\|_{0,-\frac{1}{2}}^{\frac{3}{2}}\left\|\varphi_{N}^{\varepsilon}-2 \varepsilon \tilde{R}_{o s c, N}^{\varepsilon}+2 V\right\|_{\frac{1}{2}, s} d \tau \\
& +C \int_{0}^{t}\left\|R_{o s c}^{\varepsilon, N}+\varepsilon r_{o s c, N}^{\varepsilon}\right\|_{0,-\frac{1}{2}}^{2} d \tau+C \int_{0}^{t}\left\|\varphi_{N}^{\varepsilon}\right\|_{0,-\frac{1}{2}}^{2} d \tau d \tau .
\end{aligned}
$$

Using that $a b \leq \frac{1}{4} a^{4}+\frac{3}{4} a^{\frac{4}{3}}$ for suitable choice of $a$ and $b$, we deduce that

$$
\begin{aligned}
& \left\|\varphi_{N}^{\varepsilon}\right\|_{0,-\frac{1}{2}}^{2}+2 \nu \int_{0}^{t}\left\|\nabla_{h} \varphi_{N, 1}^{\varepsilon}\right\|_{0,-\frac{1}{2}}^{2} d \tau+2 \eta \int_{0}^{t}\left\|\nabla_{h} \varphi_{N, 2}^{\varepsilon}\right\|_{0,-\frac{1}{2}}^{2} d \tau \\
\leq & 2 \min (\nu, \eta) \int_{0}^{t}\left\|\varphi_{N}^{\varepsilon}\right\|_{1,-\frac{1}{2}}^{2} d \tau+C \int_{0}^{t}\left\|R_{o s c}^{\varepsilon, N}+\varepsilon r_{o s c, N}^{\varepsilon}\right\|_{-1,-\frac{1}{2}}^{2} d \tau \\
& +C \int_{0}^{t}\left\|\varphi_{N}^{\varepsilon}\right\|_{0,-\frac{1}{2}}^{2}\left(\left\|\varphi_{N}^{\varepsilon}-2 \varepsilon \tilde{R}_{o s c, N}^{\varepsilon}+2 V\right\|_{\frac{1}{2}, s}^{4}+1\right) d \tau .
\end{aligned}
$$

About the low frequencies terms, we have the following lemma:

Lemma 4. There exists a constant $C_{N}(T)$ which depends only on $T$ and $N$ such that

$$
\left\|r_{o s c, N}^{\varepsilon}\right\|_{L_{T}^{2}\left(H^{\left.-1,-\frac{1}{2}\right)}\right.} \leq C_{N}(T)
$$

and

$$
\left\|\tilde{R}_{o s c, N}^{\varepsilon}\right\|_{L_{T}^{\infty}\left(H^{0, s}\right) \cap L_{T}^{2}\left(H^{1, s}\right)} \leq C_{N}(T) .
$$

Proof. Recall that all the functions considered here are truncated in low frequencies and the fact that

$$
\omega_{k, m, n}^{j, j^{\prime}, j^{\prime \prime}} \neq 0
$$

implies that

$$
\frac{1}{\left|\omega_{k, m, n}^{j, j^{\prime}, j^{\prime \prime}}\right|} \leq C(N) .
$$

By Lemma 2, one can conclude about $r_{o s c, N}^{\varepsilon}$. Lemma 3 with the fact that $V^{\varepsilon}$ belongs to $L_{T}^{\infty}\left(H^{0, s}\right) \cap L_{T}^{2}\left(H^{1, s}\right)$ gives the result for $\tilde{R}_{o s c, N}^{\varepsilon}$.

Since

$$
\varphi_{N}^{\varepsilon}=V^{\varepsilon}-V+R_{o s c}^{\varepsilon, N},
$$


then equation (21) becomes

$$
\begin{aligned}
& \left\|\varphi_{N}^{\varepsilon}\right\|_{0,-\frac{1}{2}}^{2}+2 \nu \int_{0}^{t}\left\|\nabla_{h} \varphi_{N, 1}^{\varepsilon}\right\|_{0,-\frac{1}{2}}^{2} d \tau+2 \eta \int_{0}^{t}\left\|\nabla_{h} \varphi_{N, 2}^{\varepsilon}\right\|_{0,-\frac{1}{2}}^{2} d \tau \\
\leq & 2 \min (\nu, \eta) \int_{0}^{t}\left\|\varphi_{N}^{\varepsilon}\right\|_{1,-\frac{1}{2}}^{2} d \tau+C \int_{0}^{t}\left\|R_{o s c}^{\varepsilon, N}+\varepsilon r_{o s c, N}^{\varepsilon}\right\|_{0,-\frac{1}{2}}^{2} d \tau \\
& +C \int_{0}^{t}\left\|\varphi_{N}^{\varepsilon}\right\|_{0,-\frac{1}{2}}^{2}\left(\left\|V^{\varepsilon}\right\|_{\frac{1}{2}, s}^{4}+\left\|V^{\varepsilon}\right\|_{\frac{1}{2}, s}^{4}+\varepsilon^{4} C(N)+1\right) d \tau .
\end{aligned}
$$

It follows that

(23) $\left\|\varphi_{N}^{\varepsilon}\right\|_{0,-\frac{1}{2}}^{2} \leq C\left\|R_{\text {osc }}^{\varepsilon, N}\right\|_{L_{T}^{2}\left(H^{0,-\frac{1}{2}}\right)}^{2}+\varepsilon^{2} C_{N}(T)+C \int_{0}^{t}\left\|\varphi_{N}^{\varepsilon}\right\|_{0,-\frac{1}{2}}^{2} h(\tau) d \tau$,

where

$$
h(\tau)=\left\|V^{\varepsilon}\right\|_{\frac{1}{2}, s}^{4}+\left\|V^{\varepsilon}\right\|_{\frac{1}{2}, s}^{4}+\varepsilon^{4} C(N)+1 .
$$

By interpolation inequality, we have

$$
\left\|V^{\varepsilon}\right\|_{\frac{1}{2}, s} \leq\left\|V^{\varepsilon}\right\|_{0, s}^{\frac{1}{2}}\left\|V^{\varepsilon}\right\|_{1, s}^{\frac{1}{2}} .
$$

Then,

$$
\int_{0}^{T}\left\|V^{\varepsilon}\right\|_{\frac{1}{2}, s}^{4}(\tau) d \tau \leq\left\|V^{\varepsilon}\right\|_{L_{T}^{\infty}\left(H^{0, s}\right)}^{2}\left\|V^{\varepsilon}\right\|_{L_{T}^{2}\left(H^{1, s}\right)}^{2} .
$$

Since the same holds for $V$, then the function $h$ belongs to $L_{T}^{1}$.

Applying Gronwall's lemma, we deduce that

$$
\begin{aligned}
\left\|\varphi_{N}^{\varepsilon}\right\|_{0,-\frac{1}{2}}^{2} \leq & \left(C\left\|R_{o s c}^{\varepsilon, N}\right\|_{L_{T}^{2}\left(H^{0,-\frac{1}{2}}\right)}^{2}+\varepsilon^{2} C_{N}(T)\right) \\
& \times \exp \left(\left(C+\varepsilon^{4} C(N)\right) T+C\left\|V^{\varepsilon}\right\|_{L_{T}^{\infty}\left(H^{0, s}\right)}\left\|V^{\varepsilon}\right\|_{L_{T}^{2}\left(H^{1, s}\right)}^{2}\right. \\
& \left.+C\|V\|_{L_{T}^{\infty}\left(H^{0, s}\right)}\|V\|_{L_{T}^{2}\left(H^{1, s}\right)}^{2}\right) .
\end{aligned}
$$

To deal with the high frequencies term $R_{o s c}^{\varepsilon, N}$, we introduce the following lemma:

Lemma 5. For any function $f \in \mathcal{C}_{T}^{0}\left(H^{0, s}\right)$ with $s \in \mathbb{R}$, the high frequency term

$$
f^{N}=\mathcal{F}^{-1}\left(1_{[N,+\infty[} \mathcal{F}(f)\right)
$$

goes to zero when $N$ goes to infinity in $\mathcal{C}_{T}^{0}\left(H^{0, s}\right)$.

Proof. Following ideas from [6] and [7], we recall that

$$
\left\|f^{N}(t)\right\|_{H^{s}}^{2}=\sum_{|k| \geq N}|k|^{2 s}|\mathcal{F}(f)(t, k)|^{2} .
$$

The desired result is due to Dini's Theorem which implies that $\left\|f^{N}(t)\right\|_{H^{s}}^{2}$ goes to zero uniformly in $t$.

This lemma implies in a straightforward way the following proposition: 
Proposition 1. The high frequency term $R_{\text {osc }}^{\varepsilon, N}$ goes to zero in $\mathcal{C}_{T}^{0}\left(H^{0, s}\right) \cap$ $L_{T}^{2}\left(H^{0,-\frac{1}{2}}\right)$, uniformly in $\varepsilon$, when $N$ goes to infinity.

Using Proposition 1 and letting $\varepsilon \rightarrow 0, N \rightarrow+\infty$, we get

$$
W^{\varepsilon} \rightarrow 0 \text { in } \mathcal{C}^{0}\left([0, T], H^{0,-\frac{1}{2}}\left(\mathbb{T}^{3}\right)\right) .
$$

An interpolation argument completes the proof.

Remark 2. Depending on which is the minimum of the couple $(\nu, \eta)$, we can conclude that if $\min (\nu, \eta)=\eta$, then

$$
W_{1}^{\varepsilon} \rightarrow 0 \text { in } L^{2}\left([0, T], H^{1,-\frac{1}{2}}\left(\mathbb{T}^{3}\right)\right)
$$

and if $\min (\nu, \eta)=\nu$, then

$$
W_{2}^{\varepsilon} \rightarrow 0 \text { in } L^{2}\left([0, T], H^{1,-\frac{1}{2}}\left(\mathbb{T}^{3}\right)\right) .
$$

\section{References}

[1] J. Benameur, S. Ibrahim, and M. Majdoub, Asymptotic study of a magneto-hydrodynamic system, Differential Integral Equations 18 (2005), no. 3, 299-324.

[2] J. Benameur and R. Selmi, Study of anisotropic MHD system in anisotropic Sobolev spaces, Ann. Fac. Sci. Toulouse Math. (6) 17 (2008), no. 1, 1-22.

[3] B. Desjardins, E. Dormy, and E. Grenier, Stability of mixed Ekman-Hartmann boundary layers, Nonlinearity 12 (1999), no. 2, 181-199.

[4] E. Dormy, Thèse de Doctorat, Institut de Physique de Globe, place Jussieu, 75005 Paris, France.

[5] E. Dormy, P. Cardin, and D. Jault, MHD flow in a slightly differantially rotating spherical shell, with conducting inner core, in a dipolar magnetic field, Earth Planet. Sci. Lett. 160 (1998), 15-30.

[6] I. Gallagher, Applications of Schochet's methods to parabolic equations, J. Math. Pures Appl. (9) 77 (1998), no. 10, 989-1054.

[7] - Asymptotic of the solutions of hyperbolic equations with a skew-symmetric perturbation, J. Differential Equations 150 (1998), no. 2, 363-384.

[8] E. Grenier, Oscillatory perturbations of the Navier-Stokes equations, J. Math. Pures Appl. (9) 76 (1997), no. 6, 477-498.

[9] D. Iftimie, The resolution of the Navier-Stokes equations in anisotropic spaces, Rev. Mat. Iberoamericana 15 (1999), no. 1, 1-36.

[10] _ A uniqueness result for the Navier-Stokes equations with vanishing vertical viscosity, SIAM J. Math. Anal. 33 (2002), no. 6, 1483-1493.

[11] J.-L. Lions, Quelques méthodes de résolution des problémes aux limites non linéaires, Dunod; Gauthier-Villars, Paris 1969.

[12] R. Selmi, Convergence results for MHD system, Int. J. Math. Math. Sci. 2006 (2006), Art. ID 28704, 19 pp.

[13] S. Schochet, Fast singular limits of hyperbolic PDEs, J. Differential Equations 114 (1994), no. 2, 476-512.

[14] R. Temam, Navier-Stokes Equations, Theory and Numerical Analysis, North Holland, Amsterdam, 1984.

DÉPARTEMEnt de Mathématique FACUlté des SCIENCES DE Gabès Gabès 6000, Tunisie

E-mail address: Ridha.Selmi@isi.rnu.tn 